\title{
BMJ Open Mapping patient pathways and estimating resource use for point of care versus standard testing and treatment of chlamydia and gonorrhoea in genitourinary medicine clinics in the UK
}

\author{
Elisabeth J Adams, ${ }^{1,2}$ Alice Ehrlich, ${ }^{1}$ Katherine M E Turner, ${ }^{2}$ Kunj Shah, ${ }^{1}$ \\ John Macleod, ${ }^{2}$ Simon Goldenberg, ${ }^{3}$ Robin K Meray, ${ }^{1}$ Vikki Pearce, ${ }^{4}$ \\ Patrick Horner ${ }^{2,5}$
}

To cite: Adams EJ, Ehrlich A, Turner KME, et al. Mapping patient pathways and estimating resource use for point of care versus standard testing and treatment of chlamydia and gonorrhoea in genitourinary medicine clinics in the UK. BMJ Open 2014;4: e005322. doi:10.1136/ bmjopen-2014-005322

- Prepublication history and additional material is available. To view please visit the journal (http://dx.doi.org/ 10.1136/bmjopen-2014005322).

Received 22 March 2014 Revised 2 July 2014 Accepted 3 July 2014

CrossMark

For numbered affiliations see end of article.

Correspondence to Dr Elisabeth J Adams; elisabeth.adams@aquariusph. com

\section{ABSTRACT}

Objectives: We aimed to explore patient pathways using a chlamydia/gonorrhoea point-of-care (POC) nucleic acid amplification test (NAAT), and estimate and compare the costs of the proposed POC pathways with the current pathways using standard laboratorybased NAAT testing.

Design/participants: Workshops were conducted with healthcare professionals at four sexual health clinics representing diverse models of care in the UK. They mapped out current pathways that used chlamydia/gonorrhoea tests, and constructed new pathways using a POC NAAT. Healthcare professionals' time was assessed in each pathway.

Outcome measure: The proposed POC pathways were then priced using a model built in Microsoft Excel, and compared to previously published costs for pathways using standard NAAT-based testing in an offsite laboratory.

Results: Pathways using a POC NAAT for asymptomatic and symptomatic patients and chlamydia/gonorrhoea-only tests were shorter and less expensive than most of the current pathways. Notably, we estimate that POC testing as part of a sexual health screen for symptomatic patients, or as stand-alone chlamydia/gonorrhoea testing, could reduce costs per patient by as much as $£ 16$ or $£ 6$, respectively. In both cases, healthcare professionals' time would be reduced by approximately 10 min per patient.

Conclusions: POC testing for chlamydia/gonorrhoea in a clinical setting may reduce costs and clinician time, and may lead to more appropriate and quicker care for patients. Further study is warranted on how to best implement POC testing in clinics, and on the broader clinical and cost implications of this technology.

\section{INTRODUCTION}

Chlamydia trachomatis and Neisseria gonorrhoea are common sexually transmitted infections,

\section{Strengths and limitations of this study}

- The main strength of this study is that it presents the first estimates of the costs of implementing chlamydia and gonorrhoea point-of-care testing compared to standard care (off-site laboratory processing of samples) in genitourinary medicine clinics, presenting a consensus from four clinics across England representing a range of service delivery models.

- The main limitation is that this is based on expert clinical opinion, rather than prospectively collected data, as point-of-care testing for chlamydia and gonorrhoea had not yet been implemented in England when this study was conducted.

- The other limitation is that we used national cost data for the inputs rather than local costs; however the care pathways could be adapted to include a Trust's costs and hence be more relevant for clinics.

and if untreated may cause pelvic inflammatory disease, which can result in serious reproductive sequelae, such as tubal factor infertility and ectopic pregnancy. ${ }^{1-3}$

Typically, the nucleic acid amplification test (NAAT) result for chlamydia and gonorrhoea is available within a week from an off-site laboratory, but it can sometimes be over 2 weeks until the patient receives treatment and some patients may not return for treatment. $^{4} 5$ If the results of these tests could be available at a single visit, outcomes could be improved by achieving earlier treatment and partner notification, minimising the risk of onward transmission and developing complications. ${ }^{6-8}$ Such a service would also likely be more convenient for patients. $^{7-9}$ 
This could be achieved with sensitive and specific NAAT point-of-care tests (POCTs), which have recently become available. One such test, Cepheid Xpert CT/ NG (Cepheid, Sunnyvale, California, USA), has at least equivalent performance to traditional laboratory-based NAATs, providing results within $90 \mathrm{~min}$ of specimen collection. $^{10}$

We have developed a model that enables us to evaluate the components of patient care pathways and associated costs. In this report we review current patient pathways in sexual health clinics that include chlamydia and gonorrhoea testing and treatment, explore and map new pathways to incorporate chlamydia and gonorrhoea POC testing efficiently in genitourinary medicine (GUM) clinics in the UK, estimate the costs of the new pathways and compare the costs with the current pathways using standard laboratory-based NAATs.

\section{METHODS}

Patient pathways using chlamydia/gonorrhoea POCTs were developed during workshops at four sexual health clinics, attended by 3-15 staff members across all clinic staff grades. Pathways were based on expert opinion delivered during the workshops, rather than any actual patient flow data. To increase applicability of the pathways across different service types, we chose a range of clinics with diverse services and service delivery. Urban and rural clinics (two in the South West of England and two in London), those providing either GUM or integrated sexual health services, and those serving higher risk populations were represented. Based on staff input, patient pathways were constructed to model the consecutive clinical steps involved in testing and treatment, for POC pathways and also current service delivery (standard care).

A model was built in Microsoft Excel to replicate pathway steps and estimate costs. We assumed the perspective of the National Health Service (NHS), and only clinical costs were included (£UK2012); patient time and associated costs were excluded. The staff cost per minute included indirect and overhead costs. Standard off-site laboratory-based chlamydia/gonorrhoea testing costs and all other cost inputs were taken from previously published sources using an identical modelling approach, ${ }^{11}$ to allow for direct comparison between current standard pathways and proposed POC pathways (see table A in the online supplementary material: file 1). POCT costs were based on the use of the only currently commercially available chlamydia/gonorrhoea POCT, the Cepheid Xpert CT/NG system, with a baseline assumed cost of $£ 18$, which includes the cartridge, machine rental, service and maintenance, assuming 15000 tests are performed annually (ie, 60 tests/day, Monday to Friday, an estimated number of tests for an average GUM in England), plus an additional £1.71 for the Cepheid sample collection materials (data from Cepheid). We varied the POCT cost from £13/test (assuming 20000 tests or more annually, ie, large clinic) to $£ 25$ (assuming one test/day, very small clinic). These costs exclude staff training (likely to be $30 \mathrm{~min}$ per person). This compares to an assumed baseline cost of $£ 12$ for the standard test plus $£ 1.35$ for the sample collection materials. We assumed that the standard test cost included all aspects of test processing including any transportation costs.

The outputs of the model were the costs of each testing pathway as a primary pathway (delivered on its own) and as an additional pathway (delivered in conjunction with other clinical services). To estimate the cost of additional pathways, staff time was weighted by the proportion of additional time, but all non-staff inputs are included at full value.

$$
\begin{aligned}
\text { The total pathway cost }= & (\text { Cost Step } 1)+(\text { Cost Step } 2) \\
& +\cdots+(\operatorname{Cost} \text { Step } N) .
\end{aligned}
$$

Detailed descriptions of patient pathway development and cost modelling are available as online supplementary materials: file 1 .

\section{RESULTS}

Patient testing pathways consist of a set of standard steps: patient registration, consultation, clinical examination, sample collection (blood, urine and/or vaginal swab), health promotion counselling, off-site laboratory-based sample processing, on-site POC testing, microscopic analysis of specimens in the clinic, results counselling, results management (data entry and notifying patients of results) and contacting patients who test positive to ensure follow-up treatment. Detailed descriptions of each step are available in table B in the online supplementary material. The standard and POC pathways differ in which steps are used, time for each and order of steps. Chlamydia and gonorrhoea testing may be carried out as either stand-alone testing or as part of a full sexual health screen including additional testing for syphilis and HIV. The sexual health screen for asymptomatic versus symptomatic patients differs in that the latter includes a clinical examination, culture for gonorrhoea and microscopy. The steps of these pathways are shown, along with costs and clinician time, in table 1A. There are two proposed sexual health screen pathways using POC testing, given as POC1 and POC2. Details of all resources used are given in the online supplementary materials: file 2 .

The lengthiest and most costly pathway for chlamydia/ gonorrhoea testing is the sexual health screen for symptomatic patients. A proposed POC pathway could reduce this cost from $£ 99$ to $£ 92$ per patient (averaging POC1 and POC2), and reduce healthcare professional time from 47 to $44 \mathrm{~min}$ per patient as a primary pathway. There was a difference in the two proposed POC pathways for symptomatic patients; namely, POC1 includes a slightly longer consultation for patients (15 vs $10 \mathrm{~min}$ ) 
Table 1 Comparison of current and proposed point-of-care clinical pathways for chlamydia and gonorrhoea testing and treatment

\begin{tabular}{|c|c|c|c|c|c|}
\hline \multirow[b]{2}{*}{ Pathway } & \multirow[b]{2}{*}{ Clinical steps } & \multicolumn{2}{|c|}{$\begin{array}{l}\text { As primary } \\
\text { pathway }\end{array}$} & \multicolumn{2}{|c|}{$\begin{array}{l}\text { As additional } \\
\text { pathway }\end{array}$} \\
\hline & & $\begin{array}{l}\text { Cost per } \\
\text { patient }\end{array}$ & $\begin{array}{l}\text { Time } \\
(\min )\end{array}$ & $\begin{array}{l}\text { Cost per } \\
\text { patient }\end{array}$ & $\begin{array}{l}\text { Time } \\
(\min )\end{array}$ \\
\hline \multicolumn{6}{|c|}{ (A) Testing pathways } \\
\hline \multicolumn{6}{|c|}{ Chlamydia and gonorrhoea only } \\
\hline Current & $\begin{array}{l}\text { Consultation } \rightarrow \text { Sample collection }{ }^{*} \rightarrow \text { Health } \\
\text { promotion } \rightarrow \text { Off-site sample processing }(1-2 \text { weeks }) \rightarrow \\
\text { Results management } \rightarrow \text { Contact positives }\end{array}$ & $£ 45.34$ & 32.8 & $£ 28.65$ & 15.7 \\
\hline POC & $\begin{array}{l}\text { Consultation } \rightarrow \text { Sample collection } \rightarrow \text { POCT }(90 \text { min }) \rightarrow \text { Results } \\
\text { management } \rightarrow \text { Contact positives }\end{array}$ & $£ 38.76$ & 21.8 & $£ 32.59$ & 13.4 \\
\hline \multicolumn{6}{|c|}{ Sexual health screen for asymptomatic patients } \\
\hline Current & $\begin{array}{l}\text { Consultation } \rightarrow \text { Sample collection }{ }^{*} \dagger \rightarrow \text { Health } \\
\text { promotion } \rightarrow \text { Off-site sample processing }(1-2 \text { weeks }) \rightarrow \\
\text { Results management } \rightarrow \text { Contact positives }\end{array}$ & $£ 79.77$ & 37.2 & $£ 54.86$ & 12.3 \\
\hline POC1 & $\begin{array}{l}\text { Consultation } \rightarrow \text { Sample collection }{ }^{*} \rightarrow \text { POCT } \\
(90 \text { min }) \rightarrow \text { Results management }(\text { POCT }) \rightarrow \text { Off-site sample } \\
\text { processing }(1-2 \text { weeks) } \rightarrow \text { Results management (HIV, } \\
\text { syphilis }) \rightarrow \text { Contact positives }\end{array}$ & $£ 77.42$ & 31.2 & $£ 69.43$ & 21.1 \\
\hline POC2 & $\begin{array}{l}\text { Sample collection } ¥ \rightarrow \text { POCT }(90 \text { min }) \rightarrow \text { Consultation/Results } \\
(\text { POCT }) \rightarrow \text { Sample collection } \dagger \rightarrow \text { Off-site sample processing } \\
(1-2 \text { weeks) } \rightarrow \text { Results management (HIV, syphilis) } \rightarrow \text { Contact } \\
\text { positives }\end{array}$ & $£ 73.57$ & 26.2 & $£ 65.57$ & 16.1 \\
\hline \multicolumn{6}{|c|}{ Sexual health screen for symptomatic patients } \\
\hline Current & $\begin{array}{l}\text { Consultation } \rightarrow \text { Exam/Sample collection }{ }^{*} \dagger \rightarrow \text { Microscopy } \rightarrow \\
\text { Health promotion } \rightarrow \text { Off-site sample processing } \\
(1-2 \text { weeks }) \rightarrow \text { Results management } \rightarrow \text { Contact positives }\end{array}$ & $£ 99.38$ & 47.2 & $£ 73.82$ & 22.3 \\
\hline POC1 & $\begin{array}{l}\text { Consultation } \rightarrow \text { Exam/Sample collection }{ }^{*} \rightarrow \text { POCT } \\
(90 \text { min }) \rightarrow \text { Microscopy } \rightarrow \text { Health promotion } \rightarrow \text { Results } \\
\text { management }(\text { POCT }) \rightarrow \text { Off-site sample processing } \\
(1-2 \text { weeks }) \rightarrow \text { Results management (HIV, syphilis) } \rightarrow \text { Contact } \\
\text { positives }\end{array}$ & $£ 100.39$ & 52.2 & $£ 74.98$ & 27.3 \\
\hline POC2 & $\begin{array}{l}\text { Sample collection }{ }^{*} \neq \rightarrow \text { POCT }(90 \mathrm{~min}) \rightarrow \text { Consultation/Results } \\
(\mathrm{POCT}) \rightarrow \text { Exam } / \text { Sample collection } \dagger \rightarrow \text { Microscopy } \rightarrow \text { Off-site } \\
\text { sample processing }(1-2 \text { weeks }) \rightarrow \text { Results management } \\
(\text { HIV, syphilis }) \rightarrow \text { Contact positives }\end{array}$ & $£ 84.46$ & 36.2 & $£ 70.72$ & 21.1 \\
\hline \multicolumn{6}{|c|}{ (B) Treatment pathways } \\
\hline Chlamydia & $\begin{array}{l}\text { Results } \rightarrow \text { Treatment } \rightarrow \text { Partner notification } \rightarrow \text { Supported partner } \\
\text { notification }\end{array}$ & $£ 34.89$ & 23.5 & $£ 24.99$ & 13.5 \\
\hline $\begin{array}{l}\text { Gonorrhoea 1st } \\
\text { visit }\end{array}$ & $\begin{array}{l}\text { Exam/Sample collection* }{ }^{*} \text { for } \mathrm{NG} \text { culture) } \rightarrow \text { Treatment } \rightarrow \text { Health } \\
\text { promotion/Partner notification } \rightarrow \text { Off-site sample } \\
\text { processing } \rightarrow \text { Supported partner notification }\end{array}$ & $£ 72.07$ & 51.0 & $£ 61.88$ & 38.0 \\
\hline $\begin{array}{l}\text { Gonorrhoea 2nd } \\
\text { visit TOC Current }\end{array}$ & $\begin{array}{l}\text { Sample collection* (for NG culture and NAAT) } \rightarrow \text { Off-site } \\
\text { sample processing (1-2 weeks) } \rightarrow \text { Results management }\end{array}$ & $£ 39.98$ & 20 & $£ 32.89$ & 10 \\
\hline $\begin{array}{l}\text { Gonorrhoea } 2 \text { nd } \\
\text { visit TOC POC }\end{array}$ & $\begin{array}{l}\text { Sample collection* (for NG culture and POCT) } \rightarrow \text { POCT } \\
(90 \text { min) } \rightarrow \text { Off-site sample processing (for NG culture, } \\
2-4 \text { days) } \rightarrow \text { Results management }\end{array}$ & $£ 45.87$ & 20 & $£ 38.77$ & 10 \\
\hline \multicolumn{6}{|c|}{$\begin{array}{l}\text { The clinical pathway steps for chlamydia and gonorrhoea (A) testing and (B) treatment are shown along with cost per patient and minutes of } \\
\text { healthcare professionals' time for each pathway when delivered as either a primary or an additional pathway. The first step, patient } \\
\text { registration, is common to all pathways and is not shown. Alternative POC pathways proposed by different clinics are reported as POC1 and } \\
\text { POC2. The cost of POC pathways may vary by - } ₹ 5 /+£ 7 \text { based on volume of tests performed. } \\
\text { *Urine/vulvo-vaginal swab collected for chlamydia and gonorrhoea testing. } \\
\text { tBlood sample collected for HIV and syphilis testing. } \\
\text { tPatients would drop off sample and book an appointment later in the day for their consultation and results. } \\
\text { NG, Neisseria gonorrhoea; NAAT, nucleic acid amplification test; POC, point-of-care; POCT, point-of-care tests; TOC, test of cure performed } \\
\text { four weeks after initial treatment for gonorrhoea. }\end{array}$} \\
\hline
\end{tabular}


and an additional step for health promotion with a health adviser for $6 \mathrm{~min}$. A portion of the cost savings arises from eliminating the need for gonorrhoea culture in symptomatic patients, except for those testing positive by POCT. Reductions in cost and time with POC testing are also expected for stand-alone chlamydia/gonorrhoea testing and for asymptomatic sexual health screen pathways, when delivered as primary pathways.

In some clinics, asymptomatic patients may be offered a rapid sexual health screen, which utilises laboratorybased chlamydia/gonorrhoea testing but reduces costs and clinician time by combining certain steps. The rapid sexual health screen is the most cost-efficient and timeefficient sexual health screen pathway (see table $\mathrm{C}$ in the online supplementary material: file 1 ) but may not be appropriate for all patients. In addition, stand-alone chlamydia/gonorrhoea self-service testing is offered in some clinics, but only to asymptomatic patients; however, incorporating a POCT into this pathway would increase costs compared to standard care (see table $\mathrm{C}$ in the online supplementary material: file 1 ).

Table 1B outlines the clinical steps and costs of treating patients for chlamydia or gonorrhoea. Pathway steps specific to treatment include gonorrhoea culture for confirmation and antimicrobial sensitivity testing, treatment with antibiotics, counselling and if requested, support/assistance with partner notification. A benefit of POC testing is that patients can initiate treatment during the same visit at which they receive positive diagnosis, eliminating the need for a follow-up treatment visit, resulting in lower overall cost. For example, under current practice, it would cost £114.66 to screen and treat a chlamydia positive patient having an asymptomatic screen (primary screen, £79.77; primary treatment at second attendance, £34.89). This could be reduced to $£ 100.49$ with a POCT, as the patient would be treated as part of the same attendance (primary screen, $£ 75.50$ (average of POC1 and POC2); additional treatment, £24.99).

Furthermore, under current practice, some symptomatic patients and partners of positive patients are treated empirically at their testing visit before laboratoryconfirmed results are available. Symptomatic patients treated presumptively for chlamydia or gonorrhoea infection incur costs of £124.37 and £201.24 per patient, respectively ( primary symptomatic sexual health screen, $£ 99.38$; plus additional chlamydia treatment, £24.99; or gonorrhoea treatment including a 4-week follow-up test of cure, £101.86). If a POCT were used on these symptomatic patients, the cost of the testing and treatment pathways (averaging POC1 and POC2) would be less than standard care (chlamydia, £117.42; gonorrhoea, $£ 200.18$ ) and the number of patients treated inappropriately would be reduced. This could enable more appropriate treatment if a non-specific genital tract infection is suspected, or potentially no treatment at all which would reduce costs by $£ 24.99$ for chlamydia and $£ 101.86$ for gonorrhoea.

\section{DISCUSSION}

Our results indicate that the total cost of most chlamydia/ gonorrhoea testing pathways in GUM clinics would be similar or reduced by using POC testing in place of off-site laboratory-based testing, and staff time would be reduced. In addition to these benefits, POC technologies have the potential to significantly improve sexual healthcare, enabling for the first time, accurate chlamydia-specific and gonorrhoea-specific diagnoses to be made and appropriately treated in a single visit. This may reduce the number of onward transmissions, inappropriate treatments in patients who are chlamydia or gonorrhoea negative but treated presumptively and prevent pelvic inflammatory disease in women. ${ }^{6}$ Interestingly, many of the proposed POC pathways could inform redesign of standard pathways without swapping to a POCT. This highlighted that many clinics could improve efficiency in service delivery even before implementing a POCT.

By reducing loss to follow-up, chlamydia/gonorrhoea POC testing could be particularly useful with groups who are less likely to return for treatment, ${ }^{2} 57$ including high-risk groups, such as men who have sex with men and commercial sex workers. Patients who do not return, or are lost to follow-up, may comprise up to $10 \%$ of all chlamydia diagnoses, ${ }^{5}$ although this may be less in GUM clinics. Chlamydia/gonorrhoea POC pathways could also be implemented in non-GUM settings, such as termination of pregnancy or contraception clinics. Women infected with chlamydia or gonorrhoea are at increased risk of developing pelvic inflammatory disease following insertion of an intrauterine device or termination of pregnancy, ${ }^{12}{ }^{13}$ and would benefit from a rapid diagnosis. ${ }^{6}$ With POCTs, novel ways of testing are imaginable, for example, POCTs could be used in outreach, with the platform situated in a mobile sexual health testing unit that travels to particular groups such as commercial sex workers.

However, as with any new technology, potential benefits must be weighed with concerns, such as how to best manage the $90 \mathrm{~min}$ delay before results are available, although this may be more of an issue for clinicians than patients. ${ }^{8}{ }^{9}$ Owing to variation in the manner in which GUM services are configured and delivered across England, the best way to implement a POC pathway may depend on the specific clinic, based on factors including size of the clinic, location (eg, rural vs urban), patient mix, staff mix and so on. For example, patients could attend in the morning to register, provide a self-collected sample, and book a slot for later in the day to see a clinician for a consultation, blood tests, results and treatment if needed. This system of having patients drop off a sample at the beginning of the day and book in to be seen later may be attractive to patients in an urban setting, where they could either go back to work/school before their appointment, or spend time in the city centre. Clinics that currently offer a slot system such as this would easily transition to such a system using a point-of-care test, and patients' treatment could be 
started on the same day of testing. This system may not work in practice, however, as patients might give a sample but would not return later.

In many rural areas it is not feasible to make two visits on the same day to the clinic since patients may have a long journey to the GUM clinic. As many clinics experience long waits to be examined and tested, it is possible that patients would be willing to wait in clinic until the results are back, so as to be treated at the same visit. Alternatively, patients could register, have a brief consultation with a clinician, have blood samples taken and lastly provide a self-collected urine or vaginal swab sample before leaving the clinic. The clinic would later notify patients of their results with positive patients returning later in the day or the next day. While it would not offer an instantaneous result, there could still be significant benefits to patients if they were treated the next day rather than waiting 1-2 weeks as under standard care. Additional benefits and concerns of $\mathrm{POC}$ pathways are presented in table $\mathrm{D}$ in the online supplementary material: file 1 .

We anticipate that POC testing may also reduce costs associated with testing and treating partners of chlamydia-positive/gonorrhoea-positive patients and improve antimicrobial stewardship, an international priority. ${ }^{1415}$ Standard practice is to offer chlamydia and gonorrhoea treatment presumptively to partners of positive patients (epidemiological treatment) when they attend for screening. ${ }^{1617}$ As the routine NAAT result is not available at the time of testing it cannot be used to inform the decision of whether treatment is actually indicated and contacts will be treated unnecessarily. Currently, the results of testing, at the time of epidemiological treatment of partners, are used for surveillance purposes and if positive, additional partner notification when indicated. If $87.9 \%$ and $85.7 \%$ of partners of chlamydiapositive and gonorrhoea-positive patients are tested for chlamydia/gonorrhoea at the same time they receive treatment ${ }^{1}$ and $36.8 \%$ and $33.2 \%$ of the partners are positive, respectively, we estimate that using a POC NAAT on partners before treatment would save $£ 19$ and $£ 62$ for partners of chlamydia-positive and gonorrhoea-positive individuals, compared to standard care. For gonorrhoea, we estimate that there would be a cost savings if the positivity is less than $93 \%$ in partners and $50 \%$ undergo chlamydia/gonorrhoea testing, as the gonorrhoea management pathway is expensive compared to testing. Hence, efficiency savings may be gained if the prevalence in partners is low, but it would be more costly to use a POC NAAT on partners first before treatment if a high proportion of partners are positive. However, both scenarios would reduce overtreatment and therefore improve antimicrobial stewardship in genitourinary medicine. ${ }^{141518}$ Caution may be warranted however, to ensure that potential positive partners are not missed if they fall outside of the window period for detecting infection.

The main limitation of the study is that pathways are based on expert clinical opinion, rather than prospectively collected data. This is because at the time the workshops were conducted, point-of-care testing for chlamydia and gonorrhoea had not yet been implemented in England. Therefore, it was not possible to validate whether the pathways generated in the workshops were indicative of what happens in actual practice. Our study proposes several novel chlamydia-testing/gonorrhoeatesting pathways using POC technologies which may reduce costs and healthcare professionals' time in GUM clinics. Although our study is based on the use of the only currently commercially available chlamydia/gonorrhoea POCT, the Cepheid Xpert CT/NG system, we anticipate that results would be applicable to other tests with similar performance characteristics. If other tests become available, their pathway costs could be estimated and compared using the same model. However, these pathways may not be relevant for POCTs that have lower performance such as some of the previous generation tests. This is a modelling study based on theoretical pathways and we did not aim to test that the pathways would work in practice or validate the steps. Once these tests are in clinics, rigorous evaluation is required in order to ensure that they deliver the promised benefits at no additional overall cost.

\section{Author affiliations}

${ }^{1}$ Aquarius Population Health Limited, Bristol, UK

${ }^{2}$ School of Social and Community Medicine, University of Bristol, Bristol, UK ${ }^{3}$ Centre for Clinical Infection \& Diagnostics Research, Guy's \& St Thomas'

NHS Foundation Trust and King's College, London, UK

${ }^{4} \mathrm{~V}$ Pearce Consulting Ltd, Hove, UK

${ }^{5}$ Department of Genitourinary Medicine, NHS Bristol, Bristol, UK

Acknowledgements The authors would like to thank the clinic leads for organising workshops and all of the staff for their participation: Bristol Sexual Health Clinic, Andrew de Burgh-Thomas and Indrajith Karunaratne-Hope House (Gloucester GUM clinic), Simon Barton and Nneka Nwokolo-56 Dean Street (Chelsea \& Westminster NHS Trust, London), Michael BradyCamberwell Sexual Health Clinic (Kings College Hospital). Thanks to Pathway Analytics for providing the input data from their model and finally to Alisha Davies, Jeff Round and Arminder Deol for useful comments on the study and the manuscript.

Contributors EJA conducted the workshops, supervised the project, planned the pathway model, analysed and interpreted the model results and drafted the article. AE helped draft the article and provided study support. KMET provided modelling and analytical support. KS built the pathway model in Excel. JM provided input on clinical pathways and service delivery. SG provided expert knowledge regarding the use of point-of-care tests from a microbiological context. RKM helped draft the article. VP provided expert advice about the sexual health pathways and commissioning/funding. PH provided clinical input on the pathways and how point-of-care tests could be implemented, interpreted model results and helped draft the manuscript. EJA and $\mathrm{PH}$ conceived the study idea and EJA, PH, KMET, JM and SG contributed to the study design. All authors critically reviewed the article for content and approved the final submitted version.

Funding Cepheid funded Aquarius Population Health to conduct the study.

Competing interests EJA has received funding from Atlas Genetics, Astra Zeneca, Bristol University, Cepheid, Gilead, Hologic the Office for Sexual Health, Pathway Analytics and St Georges University and for sexual health consultancy and lectures; PH has received funding from HEFC, BASHH, Bristol University, Imperial College London, the CPS and Hologic for consultancy, lectures, patents and providing evidence; KMET has received funding from NIHR for a personal fellowship, and from NHS Bristol Hospitals Health Trust, 
the Office for Sexual Health and NICE for consultancy. SG has received funding from Cepheid for travel and accommodation for work not related to this submission. EJA, SG, RKM, VP, Bristol University (PH and JM), AE, and KS received funding from Aquarius Population Health for this work. Cepheid provided funding to Aquarius Population Health to conduct the study and estimates of the cost of their proprietary point-of-care test. Authors are independent for the purposes of publication and Cepheid did not have any role in the writing of this paper or veto over any results published. Other similar tests are, or are soon to be commercially available. The results presented could be applicable to any other point-of-care test with similar performance, cost, and usability. We do not make any recommendation as to which test, if any, a clinic should use.

Provenance and peer review Not commissioned; externally peer reviewed.

Data sharing statement No additional data are available.

Open Access This is an Open Access article distributed in accordance with the Creative Commons Attribution Non Commercial (CC BY-NC 4.0) license, which permits others to distribute, remix, adapt, build upon this work noncommercially, and license their derivative works on different terms, provided the original work is properly cited and the use is non-commercial. See: http:// creativecommons.org/licenses/by-nc/4.0/

\section{REFERENCES}

1. Public Health England. STI Data Tables. 2012. http://www.hpa.org uk/webc/HPAwebFile/HPAweb C/1247816547927

2. Jain A, Ison CA. Chlamydia point-of-care testing: where are we now? Sex Transm Infect 2013;89:88-9.

3. CDC. Sexually transmitted diseases treatment guidelines 2010 . 17 Dec 2010. Report No: 59 http://www.cdc.gov/std/treatment/2010/ STD-Treatment-2010-RR5912.pdf

4. Medical Foundation for AIDS \& Sexual Health \& British Association for Sexual Health and HIV. Standards for the Management of Sexually Transmitted Infections (STI) 2010. 1 Jan 2010. http://www. bashh.org/documents/MF\%20BASSH\%20standards\%2018Jan\% 20 for\%20the\%20website.pdf

5. National Chlamydia Screening Programme. National Chlamydia Screening Programme Scorecard-NCSP data based on data as of 25.05.12 for the period 1st April 2011-31st March 2012. GUM data are for the period of Jan-December 2011. 2012. http://www.
chlamydiascreening.nhs.uk/ps/resources/data-tables/NCSP Scorecard_Q1-4_2011_12.pdf

6. Turner KM, Round J, Horner P, et al. An early evaluation of clinical and economic costs and benefits of implementing point of care NAAT tests for Chlamydia trachomatis and Neisseria gonorrhoea in genitourinary medicine clinics in England. Sex Transm Infect 2014:90:104-11.

7. Watchirs Smith LA, Hillman R, Ward J, et al. Point-of-care tests for the diagnosis of Neisseria gonorrhoeae infection: a systematic review of operational and performance characteristics. Sex Transm Infect 2012;89:320-6.

8. Hsieh $\mathrm{YH}$, Hogan MT, Barnes M, et al. Perceptions of an ideal point-of-care test for sexually transmitted infections-a qualitative study of focus group discussions with medical providers. PLOS ONE 2010;5:e14144.

9. Mahilum-Tapay L, Laitila V, Wawrzyniak JJ, et al. New point of care Chlamydia Rapid Test—bridging the gap between diagnosis and treatment: performance evaluation study. BMJ 2007;335:1190-4.

10. Gaydos CA, van der Pol B, Jett-Goheen M, et al. Performance of the Cepheid CT/NG Xpert Rapid PCR Test for the Detection of Chlamydia trachomatis and Neisseria gonorrhoeae. J Clin Microbiol 2013;51:1666-72.

11. Pathway Analytics. Sexual Health Tariff. http://www. pathwayanalytics.com/sexual-health/about-the-tariff (last accessed 26 Jun 2014)

12. Blackwell $\mathrm{A}$, Thomas $\mathrm{P}$, Wareham $\mathrm{K}$, et al. Health gains from screening for infection of the lower genital tract in women attending for termination of pregnancy. Lancet 1993;342:206-10.

13. Grimes DA. Intrauterine device and upper-genital-tract infection. Lancet 2000;356:1013-19.

14. Centers for Disease Control and Prevention (CDC). Antibiotic stewardship - the ultimate return on investment. 5 Nov 2013. http:// www.cdc.gov/getsmart/healthcare/learn-from-others/factsheets/ antibiotic-use.htm

15. Department of Health. Antimicrobial stewardship: 'Smart Smartthen focus'. 18 Nov 2011. https://www.gov.uk/government/uploads/ system/uploads/attachment_data/file/215308/dh_131181.pdf

16. Bignell CJ, FitzGerald M. UK national guideline for the management of gonorrhoea in adults, 2011. Int J STI AIDS 2011;22:551-47.

17. Horner PJ, Boag FC. 2006 UK national guideline for the management of genital tract infection with Chlamydia trachomatis. British Association of Sexual Health \& HIV, 2006.

18. Ison CA. Antimicrobial resistance in sexually transmitted infections in the developed world: implications for rational treatment. Curr Opin Infect Dis 2012;25:73-8. 